\title{
Effectiveness and safety of elbasvir/grazoprevir in Korean patients with hepatitis $C$ virus infection: a nationwide real-world study
}

\author{
Eun Sun Jang ${ }^{1}$, Kyung-Ah Kim², Young Seok Kim³ ${ }^{3}$ In Hee Kim ${ }^{4}$, Byung Seok Lee ${ }^{5}$, Youn Jae Lee ${ }^{6}$, \\ Woo Jin Chung ${ }^{7}$, and Sook-Hyang Jeong ${ }^{1}$
}

\begin{abstract}
${ }^{1}$ Department of Internal Medicine, Seoul National University Bundang Hospital, Seongnam; ${ }^{2}$ Department of Internal Medicine, Inje University Ilsan Paik Hospital, Goyang; ${ }^{3}$ Department of Internal Medicine, Soonchunhyang University Bucheon Hospital, Bucheon; ${ }^{4}$ Department of Internal Medicine, Jeonbuk National University Hospital, Jeonju; ${ }^{5}$ Department of Internal Medicine, Chungnam National University College of Medicine, Daejeon; ${ }^{6}$ Department of Internal Medicine, Inje University Busan Paik Hospital, Busan; ${ }^{7}$ Department of Internal Medicine, Keimyung University School of Medicine, Daegu, Korea
\end{abstract}

Received: October 28, 2019 Revised : November 28, 2019 Accepted: December 20, 2019

\section{Correspondence to}

Sook-Hyang Jeong, M.D. Department of Internal Medicine, Seoul National University Bundang Hospital, 82 Gumi-ro 173beon-gil, Bundanggu, Seongnam 13620, Korea Tel: $+82-31-787-7034$ Fax: +82-31-787-4052 E-mail:jsh@snubh.org https://orcid.org/0000-00024916-7990
Background/Aim: This study aimed to establish the real-world effectiveness and safety of grazoprevir/elbasvir (EBR/GZR) therapy in South Korea.

Methods: A total of 242 patients with chronic hepatitis C virus (HCV) genotype 1 or 4 infection who started EBR/GZR were consecutively enrolled from seven tertiary hospitals. Retrospective analysis of the fractions of patients that achieved sustained virological response (SVR) was performed, and the incidence of adverse events was noted.

Results: The mean age of enrolled patients was $59.0 \pm 12.6$ years and $47.5 \%$ were males. Patients with HCV genotype $1 \mathrm{~b}$ accounted for $93.8 \%(\mathrm{n}=227)$, and patients with HCV of unspecified genotype 1 accounted for $5.8 \%(n=14)$. Hypertension was the most common comorbid disease $(29.8 \%)$ followed by diabetes $(22.7 \%)$ and chronic kidney disease (CKD, 12.4\%). SVR rates of treatment-naïve and treatment-experienced patients were 85.5\% (182/213) and 93.1\% (27/29), respectively, in the intention-to-treat analyses, whereas in the per-protocol analyses, those were $97.8 \%(179 / 183)$ and $100 \%(28 / 28)$, respectively. Fewer patients with HCV genotype 1 of unspecified subtype achieved SVR $(81.8 \%, \mathrm{n}=11)$ compared to the patients with SVR infected with genotype $1 \mathrm{~b}(99 \%, \mathrm{n}=198, \mathrm{p}<0.001)$. All patients with CKD showed SVR. Itching (12\%) and dyspepsia (4.1\%) were common adverse events. Of the four patients who discontinued the antiviral therapy, one experienced mild fatigue but neither treatment withdrawal was because of an adverse event.

Conclusions: EBR/GZR showed high real-world effectiveness and safety in Korean patients with chronic HCV infection regardless of the previous antiviral treatment, liver cirrhosis, or CKD status.

Keywords: Hepatitis C; Antiviral agents; Elbasvir-grazoprevir drug combination

\section{INTRODUCTION}

Hepatitis $\mathrm{C}$ virus (HCV) is an important cause of chronic liver disease and hepatocellular carcinoma (HCC). Over
71 million people are estimated to be infected worldwide, and there were 475,000 HCV-related deaths in 2015 [1-3]. In Korea, the prevalence of anti-HCV positivity has been reported to be $0.64 \%$ to $0.78 \%$ [4]; it increases with 
age and is the second most common cause of liver cirrhosis (LC) and HCC [5].

A single pill containing elbasvir (EBR) and grazoprevir (GZR), the inhibitors of non-structural proteins (NS) $5 \mathrm{~A}$ and $\mathrm{NS}_{3} / 4 \mathrm{~A}$, has been developed to control HCV infection with once daily dosing. Since May 2017, EBR/GZR combination has been approved in South Korea for the treatment of adult chronic hepatitis $\mathrm{C}$ or compensated cirrhotic patients who are infected with HCV genotype 1 or 4 . Previous clinical trials [6-8] showed that $95 \%$ to $97.8 \%$ of treatment-naïve and $88.9 \%$ of treatment-experienced patients had successfully achieved sustained virological response (SVR) in 12 weeks after completion of therapy. The treatment showed favorable efficacy in LC patients: $97 \%$ to $98 \%$ developed SVR $[6,7]$. Although race may affect the treatment response to direct-acting antiviral agents $[9,10]$, most studies from Western countries where HCV is prevalent included only a limited number of Asian patients. Recent pooled analyses of Asian participants of EBR/GZR phase 2/3 trials showed high efficacy, with $96.9 \%$ achieving SVR in 15 countries [11]. Despite $98.6 \%$ (73/74) of Korean genotype $1 \mathrm{~b}$ patients have been shown to achieve SVR12 [12], there have been no real-world data from Korean patients published so far.

Thus, we aimed to investigate the effectiveness and safety of the EBR/GZR therapy in a real-world setting in South Korea. Additionally, we sought to establish whether any comorbid diseases affected the attainment of SVR in Korean patients.

\section{METHODS}

\section{Subjects}

This retrospective study consecutively included 242 patients with HCV genotype 1 or 4 infection who received at least one dose of EBR/GZR therapy between May 2017 and June 2018. Seven tertiary hospitals located in different regions of South Korea had participated. EBR/GZR was administered orally at a fixed dose of $50 \mathrm{mg}$ of EBR and $100 \mathrm{mg}$ of GZR once daily according to the label. This study was approved by the Institutional Review Boards of the seven participating hospitals (i.e., Seoul National University Bundang Hospital, B-1809/495-108), and the requirement for obtaining written informed consent was waived because this study was based on the retrospective review of existing medical records.

Patients who had been treated with at least one dose of interferon/peginterferon-based therapy or direct-acting antiviral agents other than EBR/GZR were considered as treatment-experienced group, whereas all other patients were considered as treatment-naïve.

The persistence of HCV RNA for more than 6 months was defined as chronic hepatitis C. The diagnosis of LC was made by histologic or clinical findings of portal hypertension [13], including cirrhotic appearance of the liver with splenomegaly, revealed by imaging (ultrasonography, computed tomography, or magnetic resonance imaging), thrombocytopenia, presence of varices on endoscopy, ascites, and/or hepatic encephalopathy. Patients with hepatocellular carcinoma were considered as a separate HCC group, regardless of their liver function.

\section{Laboratory tests}

Estimated glomerular filtration rate (eGFR) was calculated using the original Modification of Diet in Renal Disease Study equation [14] based on creatinine and patient characteristics. Chronic kidney disease (CKD) was categorized into five stages according to eGFR values.

HCV genotyping and RNA concentration measurement by real-time polymerase chain reaction were performed at the certified laboratories at each participating site. In several patents with HCV genotype $1 b$, the presence of NS5A resistance-associated substitutions (RAS) was tested by direct Sanger sequencing at a central laboratory (Seoul Clinical Laboratories, Yongin, Korea). The results were reported as negative, positive, or "undetermined," based on the presence of $\mathrm{L}_{31}$ or Y93 RAS. The limit of HCV RNA concentration for the detection of RAS was $1,000 \mathrm{IU} / \mathrm{mL}$.

\section{Measurements of the treatment response and ad- verse events of antiviral therapy}

SVR was defined as undetectable HCV RNA level at 12 weeks after completion of treatment according to the practice guideline for hepatitis C management [15]. Viral breakthrough referred to the reappearance of HCV RNA during the treatment, and relapse was defined as the reappearance of HCV RNA after initial viral elimination with undetectable HCV RNA level at the end of treatment. Because several patients did not visit the clinic 
Table 1. Baseline characteristics of the studied cohort $(n=242)$

\begin{tabular}{|c|c|}
\hline Characteristic & Value \\
\hline Age, yr-old & $59.0 \pm 12.6$ \\
\hline Male sex & $111(47 \cdot 5)$ \\
\hline \multicolumn{2}{|l|}{ Liver disease status } \\
\hline Chronic hepatitis C & $169(69.8)$ \\
\hline Liver cirrhosis, compensated & $71(29 \cdot 3)$ \\
\hline Hepatocellular carcinoma & $2(0.8)$ \\
\hline \multicolumn{2}{|l|}{ Previous antiviral treatment } \\
\hline Naïve & $213(88.0)$ \\
\hline Experienced & $29(12.0)$ \\
\hline Interferon-based & $27(93.1)$ \\
\hline $\begin{array}{l}\text { Direct-acting antiviral } \\
\text { agent-based }\end{array}$ & $2(6.9)$ \\
\hline \multicolumn{2}{|l|}{ Virological study } \\
\hline RNA viral load, IU/mL & $1.4 \times 10^{6}\left(100-2.2 \times 10^{7}\right)$ \\
\hline$>800,000$ & $159(65 \cdot 7)$ \\
\hline$>2,000,000$ & $107(44.2)$ \\
\hline \multicolumn{2}{|l|}{ Genotype } \\
\hline $1 \mathrm{~b}$ & $227(93.8)$ \\
\hline 1, unspecified & $14(5.8)$ \\
\hline 4 & $1(0.4)$ \\
\hline \multicolumn{2}{|l|}{$\begin{array}{l}\text { Resistance-associated } \\
\mathrm{NS}_{5} \mathrm{~A} \text { substitutions }\end{array}$} \\
\hline Not tested & $201(83.1)$ \\
\hline Tested & $\begin{array}{l}41(16.9), \\
\text { all genotype } 1 b\end{array}$ \\
\hline Negative & $18(43.9)$ \\
\hline Positive & $24(56.1)$ \\
\hline $\mathrm{L}_{31}$ & $1(4.2)$ \\
\hline $\mathrm{L}_{31}+\mathrm{Y}_{93}$ & $15(62.5)$ \\
\hline$Y_{93}$ & $7(29.2)$ \\
\hline Not specified & $1(4 \cdot 2)$ \\
\hline HBV coinfection & $3(1.2)$ \\
\hline \multicolumn{2}{|l|}{ Laboratory findings } \\
\hline $\mathrm{WBC}, / \mathrm{mm}^{3}$ & $5,410(1,023-15,920)$ \\
\hline $\mathrm{Hb}, \mathrm{g} / \mathrm{dL}$ & $13 \cdot 4(7.1-18)$ \\
\hline $\mathrm{PLT}, \times 10^{3} / \mathrm{mm}^{3}$ & $176(38-457)$ \\
\hline $\mathrm{Cr}, \mathrm{mg} / \mathrm{dL}(\mathrm{n}=223)$ & $0.8(0.4-12.3)$ \\
\hline MDRD GFR, $\mathrm{mL} / \mathrm{min} / 1.73 \mathrm{~m}^{2}$ & $93\left(4 \cdot 5^{-191)}\right.$ \\
\hline $30-59$ & $9(3.7)$ \\
\hline $15-29$ & $1(0.4)$ \\
\hline$<15$ & $20(8.3)$ \\
\hline Albumin, g/dL & $4.4(2.2-5.2)$ \\
\hline
\end{tabular}

Table 1. Continued

\begin{tabular}{lc}
\hline Characteristic & Value \\
\hline Bilirubin, mg/dL & $0.7(0.2-7.4)$ \\
ALP, IU/L & $95(29-459)$ \\
AST, IU/L & $43(12-307)$ \\
ALT, IU/L & $38(7-603)$ \\
GGT, IU/L & $40(8-678)$ \\
PT, INR & $1.05(0.86-3.04)$ \\
\hline
\end{tabular}

Values are presented as mean $\pm \mathrm{SD}$, number (\%), or median (range).

HBV, hepatitis B virus; WBC, white blood cell; $\mathrm{Hb}$, hemoglobin; PLT, platelet; Cr, creatinine; MDRD, Modification of Diet in Renal Disease Study; ALP, alkaline phosphatase; AST, aspartate aminotransferase; ALT; alanine aminotransferase; LC, liver cirrhosis; GGT, gamma glutamyl transferase; PT, prothrombin time; INR, international normalized ratio.

for SVR evaluation at the exact weeks, the RNA data obtained in a window period of \pm 4 weeks were accepted. Patients lost to follow-up after the completion of antiviral therapy and not evaluated for SVR were considered as "no SVR" in the intention-to-treat (ITT) analysis.

The adverse events were based on medical chart review by the attending physician. Owing to the limitation of the study's retrospective design, the grade of adverse events could not be evaluated.

\section{Statistical analysis}

Analyses were conducted by descriptive statistics for virological outcomes, safety, and comorbid diseases using the chi-squared test. SVR in the ITT analysis was calculated for all enrolled patients $(n=242)$. In the per-protocol (PP) analysis, SVR was calculated for the subjects who completed the scheduled treatment and follow-up for $\geq 12$ weeks after treatment completion $(n=213)$. All statistical analyses were performed using Stata version 14.0 (Stata, College Station, TX, USA).

\section{RESULTS}

\section{Baseline patient characteristics}

As shown in Table 1, the mean age of the participants was 59.0 years (SD, 12.6), and 111 (47.5\%) were male. Most 


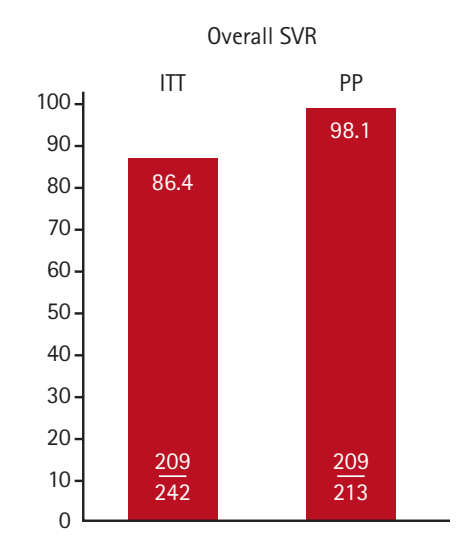

A

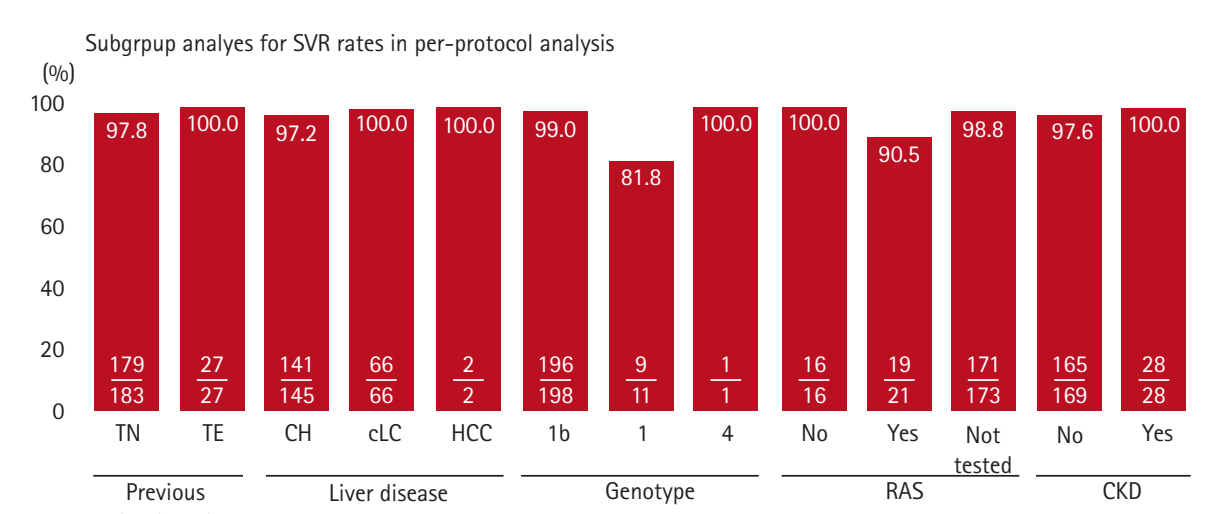

B

Figure 1. Sustained virological response (SVR) rates in response to elbasvir/grazoprevir therapy in Korean patients infected with genotype 1 or 4 hepatitis C virus. (A) Overall, (B) subgroup analyses. ITT, intention-to-treat; PP, per-protocol; TN, treatment-naïve; TE, treatment-experienced; $\mathrm{CH}$, chronic hepatitis; cLC, compensated liver cirrhosis; HCC, hepatocellular carcinoma; RAS, resistance-associated substitution; CKD, chronic kidney disease (estimated glomerular filtration rate < 60 mL/ $\left.\min / 1.73 \mathrm{~m}^{2}\right)$.

patients had chronic hepatitis (69.8\%), and 29.3\% had compensated LC. A total of 213 patients (88.0\%) were naïve for antiviral treatment, and 29 (12.0\%) had experience of other antiviral therapy before the EBR/GZR therapy (Table 1). Only one patient was infected with HCV genotype 4, and most enrolled patients had HCV genotype $1 \mathrm{~b}$, which is the major genotype in Korea. HCV genotype 1a cases were not included in this study. Forty-one patients with HCV genotype $1 \mathrm{~b}$ were screened in the RAS test, and 24 patients were found positive for NS5A RAS: one (for L31), 15 (for L31 + Y93), seven (for Y93), and one (for an undetermined RAS).

\section{SVR rates after EBR/GRZ therapy in Korean HCV-in- fected patients}

As shown in Fig. 1A, SVR was achieved by $86.4 \%$ and 98.1\% Korean patients on EBR/GRZ therapy according to the ITT and PP analyses, respectively. Of the 29 patients who were excluded from the PP analysis, four had stopped antiviral therapy within 30 days of treatment for the reasons unrelated to an adverse event or inadequate response, and 25 had completed the scheduled therapy for 12 weeks but did not visit the clinic for SVR evaluation. According to subgroup analyses (Fig. 1B), 97.8\% of treatment-naïve and 100\% of treatment-experienced patients developed SVR in the PP analysis $(p=0.442)$. We looked for the effect of the underlying liver disease and found that $100 \%$ of compensated LC patients and $97.2 \%$ of chronic hepatitis patients showed SVR $(p=0.159)$. Two HCC patients who had been treated and not recurred for more than 6 months also achieved SVR. Although their number was small, fewer patients with HCV genotype 1 of unspecified subtype achieved SVR (81.2\%) compared to the fraction of those with HCV genotype $1 \mathrm{~b}(99 \%)$. Notably, two genotype $\mathrm{ib}$ patients who failed to achieve SVR had Y93 substitution. Therefore, genotype and RAS were important predictive factors for SVR following EBR/GRZ therapy. Detailed clinical characteristics of the four patients who did not show SVR after EBR/GRZ therapy are described in Table 2.

\section{Effects of comorbid diseases on the SVR after EBR/ GRZ therapy in Korean HCV-infected patients}

In the $242 \mathrm{HCV}$-infected patients, the most common comorbid diseases were hypertension (29.8\%) and diabetes mellitus (22.7\%) (Table 3). Moderate or severe renal disease, i.e., CKD stage $\geq 3$ (eGFR $<60 \mathrm{~mL} / \mathrm{min} / 1.73 \mathrm{~m}^{2}$ ) was found in $30(12.4 \%)$ patients. Of them, 20 had stage 5 CKD (eGFR < $\left.15 \mathrm{~mL} / \mathrm{min} / 1.73 \mathrm{~m}^{2}\right)$. All CKD patients had completed 12 weeks of EBR/GZR therapy and achieved SVR according to the PP analysis. Fifteen patients had a history of hepatic or extrahepatic malignancy (6.2\%), and cerebrovascular diseases were found in 5.0\%. Of the six patients with depression, one failed to achieve SVR 
Table 2. Clinical characteristics of the four patients who failed to achieve sustained virological response after elbasvir/grazoprevir therapy

\begin{tabular}{|c|c|c|c|c|c|c|c|c|c|}
\hline Case no. & Age, yr & Sex & $\mathrm{LC}$ & $\begin{array}{l}\text { Previous } \\
\text { treatment }\end{array}$ & Genotype & $\begin{array}{c}\text { Viral load, } \\
\text { IU/mL }\end{array}$ & RAS & Comorbidities & $\begin{array}{l}\text { Treatment } \\
\text { completion }\end{array}$ \\
\hline 1 & 83 & Male & No & No & $1 b$ & $5,510,656$ & $\mathrm{Y}_{93}$ & CVA, DM & Yes \\
\hline 2 & 48 & Female & No & No & 1 & $1,190,000$ & NA & Depression & Yes \\
\hline 3 & 62 & Female & No & No & $1 b$ & $2,683,800$ & $\mathrm{Y}_{93}$ & No & Yes \\
\hline 4 & 51 & Female & No & No & 1 & $6,359,136$ & NA & No & Yes \\
\hline
\end{tabular}

LC, liver cirrhosis; RAS, resistance-associated substitutions; CVA, cerebrovascular accidents; DM, diabetes mellitus; NA, not available.

despite the completion of EBR/GZR therapy. Other comorbid diseases were not frequently found in our study cohort, and all individuals with those diseases achieved SVR.

\section{Tolerability of elbasvir/grazoprevir therapy in Korean HCV-infected patients}

Adverse events of any grade were reported in $24.4 \%$ (59/242) of patients during EBR/GZR therapy (Table 4). Itching was the most common symptom found in $12 \%$ of patients. Alanine aminotransferase (ALT) elevation greater than five times the upper limit of normal (ULN) was found in one (0.4\%) patient at week 12, whereas ALT elevation greater than $2 \times$ ULN was detected in 11 (4.5\%) patients. In three of these patients (27.3\%), ALT elevation was detected within 4 weeks after the start of antiviral treatment. In five patients (45.5\%), it was found on week 8 , and in another three patients (27.3\%)—-towards the end of the 12 weeks of treatment (Supplementary Table 1). All of these patients, except one lost to follow-up, achieved SVR successfully after 12 weeks of antiviral therapy, and 8 of 11 patients (72.7\%) showed improved ALT level without any specific treatment.

Dyspepsia, fatigue, epigastric pain, nausea was found in $2-4 \%$ of the patients (Table 4 ). Nonetheless, no subject had considered to withdraw from EBZ/GZR therapy because of the symptoms. Of the four patients who stopped antiviral therapy within 30 days in our study, three did not report any symptoms and one (54-year-old woman) felt mild fatigue during antiviral treatment.

\section{DISCUSSION}

In this retrospective real-world study in Korean patients infected with HCV genotype 1 or 4, EBR/GZR therapy proved to be highly effective with $98.1 \%$ of patients having achieved overall SVR irrespective of the previous antiviral treatment experience or presence of LC. Furthermore, EBR/GZR therapy was well tolerated in our study cohort, which included patients with various comorbid diseases, including CKD. To the best of our knowledge, this is the first real-world study of EBR/GRZ therapy in the Korean population.

A recent integrated study of Korean patients who participated in EBR/GZR clinical trials showed that all genotype $1 b$ patients who had completed EBR/GZR therapy achieved successful SVR12 [12]. Of them, 22\% had RAS. In the present study, the SVR rate was slightly lower than that in the integrated study because two genotype $1 b$ treatment-naïve patients with $\mathrm{Y}_{93}$ substitution failed to develop SVR. RAS was an important predictor of the EBR/GZR therapy efficacy in a previous real-world study from Japan. Mashiba et al. [16] had reported that NS5A L31/Y93 double mutation was found in treatment-naïve patients who failed to achieve SVR after EBR/GZR therapy. In our study, RAS testing was performed based on the commercial laboratory test only for the common sites, so patients who failed to achieve SVR after EBR/ GZR therapy may have had uncommon RAS.

The effect of HCV genotype 1 subtype on the antiviral efficacy has not been conclusively elucidated yet because the majority of clinical trials included genotype 1a or $1 b$ patients only or analyzed genotype 1 data in subtype-unspecific way, when data from all subtypes were pooled [17-19]. Notably, we found that a lower fraction of patients with HCV genotype 1 with unspecified subtype achieved SVR compared to the fraction of patients with defined HCV subtype. Because the "unspecified" result might imply genetic diversity of the virus, our data sug- 
Table 3. SVR rates with grazoprevir/elbasvir therapy in patients with hepatitis $\mathrm{C}$ virus infection and other comorbid diseases

\begin{tabular}{lcc}
\hline Variable & No. (\%) & $\begin{array}{c}\text { SVR } \\
\text { rate, \% }\end{array}$ \\
\hline Any comorbid disease & $116(47.9)$ & 98.2 \\
Hypertension & $72(29.8)$ & 100 \\
\hline Diabetes mellitus, uncomplicated & $41(16.9)$ & 100 \\
Diabetes mellitus, complicated & $14(5.8)$ & 92.3 \\
\hline Moderate or severe renal disease & $30(12.4)$ & 100 \\
Malignancy & $15(6.2)$ & 100 \\
Cerebrovascular diseases & $12(5.0)$ & 90.9 \\
\hline Depression & $6(2.5)$ & 83.3 \\
Myocardial infarction & $4(1.7)$ & 100 \\
Chronic pulmonary disease & $4(1.7)$ & 100 \\
\hline Peptic ulcer disease & $3(1.3)$ & 100 \\
\hline Peripheral vascular disease & $3(1.3)$ & 100 \\
\hline Connective tissue disease & $3(1.3)$ & 100 \\
\hline Dementia & $2(0.8)$ & 100 \\
\hline Congestive heart failure & $1(0.4)$ & 100 \\
\hline
\end{tabular}

SVR, sustained virological response.

gested that a detailed re-evaluation could be necessary in patients with the unspecified HCV genotype subtype. The phylogenetic analysis of HCV is still developing, and several novel subtypes have been described in a recent study [20]. Thus, infection with HCV genotype 1 strains of unspecified subtype may be better treated with a carefully controlled pan-genotypic antiviral regimen.

The prevalence of HCV infection is characteristically increasing with age in Korea. According to a recent nationwide study, anti-HCV seroprevalence rates in individuals over $40,50,60$, and 70 years of age were $0.38 \%$, $0.61 \%, 1.06 \%$, and $1.63 \%$, respectively [21]. Because the aged HCV-infected population must have various comorbidities, the effect of comorbid disease and concomitant medication on the antiviral efficacy is an important issue. In our study, $47.9 \%$ of studied patients had more than one comorbid disease. Nevertheless, the antiviral efficacy was not significantly influenced by the presence of comorbid diseases (Tables 2 and 3 ). In particular, $100 \%$ of patients with renal disease achieved SVR, and a previous real-world study that included patients with CKD $[22,23]$ showed that $96-98 \%$ of them developed
Table 4. Adverse events during elbasvir/grazoprevir therapy in Korean patients with hepatitis $C$ virus infection $(n=242)$

\begin{tabular}{lc}
\hline Variable & No. $(\%)$ \\
\hline Any & $59(24.4)$ \\
Itching & $29(12.0)$ \\
ALT elevation & \\
$\quad>\times 2$ ULN & $11(4.5)$ \\
$\quad>\times 5$ ULN & $1(0.4)$ \\
Dyspepsia & $10(4.1)$ \\
Fatigue & $7(2.9)$ \\
Epigastric pain & $6(2.5)$ \\
Nausea & $5(2.1)$ \\
Dizziness & $4(1.7)$ \\
Vomiting & $2(0.8)$ \\
Flu-like symptoms & $2(0.8)$ \\
Rash & $1(0.4)$ \\
Anemia & $1(0.4)$ \\
Depression & 0 \\
Others & $11(4.6)$ \\
\hline ALT alanine aminotransfras
\end{tabular}

ALT, alanine aminotransferase; ULN, upper limit of normal.

SVR. With respect to the tolerability, the frequency of adverse events in patients with CKD (18.8\%) was similar to that in patients with preserved renal function $(21.5 \%$, $p=0.794$, data not shown).

In previous clinical trials, EBR/GZR combination was well tolerated but the most common adverse events were fatigue ( $7 \%$ to $23 \%$ ), headache (10\% to $20 \%$ ), and nausea $(5 \%$ to $8 \%)[8,24,25]$. In the present study, the frequency of fatigue or headache was quite low $(<3 \%)$, but itching was reported by $12 \%$ of the patients. Because adverse events were reviewed retrospectively in our study, the symptoms might be underestimated. Nonetheless, our data suggested that clinicians who treat HCV patients need to consider itching as a common side effect of antiviral treatment, which has not been reported in clinical trials. ALT elevation over $5 \times$ ULN, which was seen in $1 \%$ of clinical trial participants according to the US FDA label [26], was observed only once in our study, whereas mild ALT elevation was found in $4.5 \%$ of subjects but not in a clear relation with antiviral treatment response. Although in the majority of patients ALT elevation subsided without any specific treatment, the detailed mech- 
anism of this phenomenon needs to be elucidated.

Our study had several limitations. First, $11.9 \%$ of the patients have not been evaluated for the SVR, so the proportion of patients achieving SVR may be either overestimated in the PP analysis or underestimated in the ITT analysis. It was an unavoidable situation in the real-world setting, but antiviral efficacy would be favorable because even three of the four patients who withdrew EBR/GRZ therapy within 30 days did not show detectable HCV RNA level at 12 weeks after antiviral therapy (data not shown). Secondly, the adverse events were not graded owing to retrospective design. Nonetheless, visits to respective physicians on each site confirmed that all treatment withdrawal cases were not related to adverse events: one person withdrew due to unaffordability and three were lost to follow-up.

In conclusion, EBR/GRZ therapy showed high real-world effectiveness and good tolerability except that itching was found in $12 \%$ of Korean patients with chronic HCV infection regardless of previous antiviral treatment, LC, or CKD.

\section{KEY MESSAGE}

1. Elbasvir/grazoprevir therapy showed high real-world effectiveness and tolerability in Korean patients with chronic hepatitis $\mathrm{C}$ virus (HCV) infection regardless of previous antiviral treatment history or presence of liver cirrhosis or chronic kidney disease.

2. Patients with HCV genotype 1 with unspecified subtype showed lower sustained virological response rate compared to those with defined subtype.

\section{Conflict of interest}

No potential conflict of interest relevant to this article was reported.

\section{Acknowledgments}

The authors are grateful to the devoted research coordinators (Hyeon Noh, Dawoon Jeong, Seon Young Park, Hyoyoung Kang, Semi Jeon, Dalli Nam, Su Jin Lee, and Young Soon Lee).

This study was funded by MSD and a grant for the Chronic Infectious Disease Cohort Study (Korea HCV
Cohort Study, 4800-4859-304) from the Korea Centers for Disease Control and Prevention.

\section{REFERENCES}

1. Franco E, Pettinicchio V, Zorzoli E. The evolution of the burden of viral hepatitis from 1990 to 2013: still an open challenge to global public health policy. Hepatobiliary Surg Nutr 2017;6:277-279.

2. Stanaway JD, Flaxman AD, Naghavi M, et al. The global burden of viral hepatitis from 1990 to 2013: findings from the Global Burden of Disease Study 2013. Lancet 2016;388:1081-1088.

3. Heffernan A, Cooke GS, Nayagam S, Thursz M, Hallett TB. Scaling up prevention and treatment towards the elimination of hepatitis C: a global mathematical model. Lancet 2019;393:1319-1329.

4. Kim DY, Kim IH, Jeong SH, et al. A nationwide seroepidemiology of hepatitis C virus infection in South Korea. Liver Int 2013;33:586-594.

5. Lee SS, Byoun YS, Jeong SH, et al. Type and cause of liver disease in Korea: single-center experience, 2005-2010. Clin Mol Hepatol 2012;18:309-315.

6. Jacobson IM, Lawitz E, Kwo PY, et al. Safety and efficacy of elbasvir/grazoprevir in patients with hepatitis $\mathrm{C}$ virus infection and compensated cirrhosis: an integrated analysis. Gastroenterology 2017;152:1372-1382.

7. Zeuzem S, Ghalib R, Reddy KR, et al. Grazoprevir-elbasvir combination therapy for treatment-naive cirrhotic and noncirrhotic patients with chronic hepatitis $\mathrm{C}$ virus genotype 1, 4, or 6 infection: a randomized trial. Ann Intern Med 2015;163:1-13.

8. Sulkowski M, Hezode C, Gerstoft J, et al. Efficacy and safety of 8 weeks versus 12 weeks of treatment with grazoprevir (MK-5172) and elbasvir (MK-8742) with or without ribavirin in patients with hepatitis $\mathrm{C}$ virus genotype 1 mono-infection and HIV/hepatitis $\mathrm{C}$ virus co-infection (C-WORTHY): a randomised, open-label phase 2 trial. Lancet 2015;385:1087-1097.

9. Su F, Green PK, Berry K, Ioannou GN. The association between race/ethnicity and the effectiveness of direct antiviral agents for hepatitis $\mathrm{C}$ virus infection. Hepatology 2017;65:426-438.

10. Benhammou JN, Dong TS, May FP, et al. Race affects SVR12 in a large and ethnically diverse hepatitis C-in- 
fected patient population following treatment with direct-acting antivirals: analysis of a single-center Department of Veterans Affairs cohort. Pharmacol Res Perspect 2018;6:000379.

11. Wei L, Kumada H, Perumalswami PV, et al. Safety and efficacy of elbasvir/grazoprevir in Asian participants with hepatitis $\mathrm{C}$ virus genotypes 1 and 4 infection. J Gastroenterol Hepatol 2019;34:1597-1603.

12. Lee YJ, Heo J, Kim DY, et al. An integrated analysis of elbasvir/grazoprevir in Korean patients with hepatitis C virus genotype $\mathrm{lb}$ infection. Clin Mol Hepatol 2019;25:400407.

13. Suk KT, Baik SK, Yoon JH, et al. Revision and update on clinical practice guideline for liver cirrhosis. Korean J Hepatol 2012;18:1-21.

14. Levey AS, Bosch JP, Lewis JB, Greene T, Rogers N, Roth D. A more accurate method to estimate glomerular filtration rate from serum creatinine: a new prediction equation. Modification of Diet in Renal Disease Study Group. Ann Intern Med 1999;130:461-470.

15. Korean Association for the Study of the Liver (KASL). 2017 KASL clinical practice guidelines management of hepatitis C: treatment of chronic hepatitis C. Clin Mol Hepatol 2018;24:169-229.

16. Mashiba T, Joko K, Kurosaki M, et al. Real-world efficacy of elbasvir and grazoprevir for hepatitis $\mathrm{C}$ virus (genotype 1): a nationwide, multicenter study by the Japanese Red Cross Hospital Liver Study Group. Hepatol Res 2019;49:1114-1120.

17. Jacobson IM, Poordad F, Firpi-Morell R, et al. Elbasvir/ Grazoprevir in people with hepatitis C genotype 1 infection and child-pugh class B cirrhosis: the C-SALT Study. Clin Transl Gastroenterol 2019;10:e00007.

18. Hernandez-Conde M, Fernandez I, Perello C, et al. Effectiveness and safety of elbasvir/grazoprevir therapy in patients with chronic HCV infection: results from the Spanish HEPA-C real-world cohort. J Viral Hepat 2019;26:55-64.
19. Zamor PJ, Vierling J, Ghalib R, et al. Elbasvir/grazoprevir in black adults with hepatitis $\mathrm{C}$ virus infection: a pooled analysis of phase 2/3 clinical trials. Am J Gastroenterol 2018;113:863-871.

20. Hedskog C, Parhy B, Chang S, et al. Identification of 19 novel hepatitis $\mathrm{C}$ virus subtypes-further expanding $\mathrm{HCV}$ classification. Open Forum Infect Dis 2019;6:ofzo76.

21. Jang ES, Ki M, Choi HY, Kim KA, Jeong SH; Korean Hepatitis Epidemiology Study Group. The change in the nationwide seroprevalence of hepatitis $C$ virus and the status of linkage to care in South Korea from 2009 to 2015. Hepatol Int 2019;13:599-608.

22. Ogawa E, Furusyo N, Azuma K, et al. Elbasvir plus grazoprevir for patients with chronic hepatitis $\mathrm{C}$ genotype 1 : a multicenter, real-world cohort study focusing on chronic kidney disease. Antiviral Res 2018;159:143-152.

23. Kramer JR, Puenpatom A, Erickson KF, et al. Real-world effectiveness of elbasvir/grazoprevir In HCV-infected patients in the US veterans affairs healthcare system. J Viral Hepat 2018;25:1270-1279.

24. Kumada H, Suzuki Y, Karino Y, et al. The combination of elbasvir and grazoprevir for the treatment of chronic HCV infection in Japanese patients: a randomized phase II/III study. J Gastroenterol 2017;52:520-533.

25. Lawitz E, Gane E, Pearlman B, et al. Efficacy and safety of 12 weeks versus 18 weeks of treatment with grazoprevir (MK-5172) and elbasvir (MK-8742) with or without ribavirin for hepatitis $\mathrm{C}$ virus genotype 1 infection in previously untreated patients with cirrhosis and patientswith previous null response with or without cirrhosis (C-WORTHY): a randomised, open-label phase 2 trial. Lancet 2015;385:1075-1086.

26. U.S. Food and Drug Administration. Product Information. Zepatier (elbasvir-grazoprevir) [Internet]. Silver Spring (MD): FDA, 2017 [cited 2020 May 2]. Available from: https://www.accessdata.fda.gov/drugsatfda_docs/ label/2017/208261soo2lbl.pdf. 


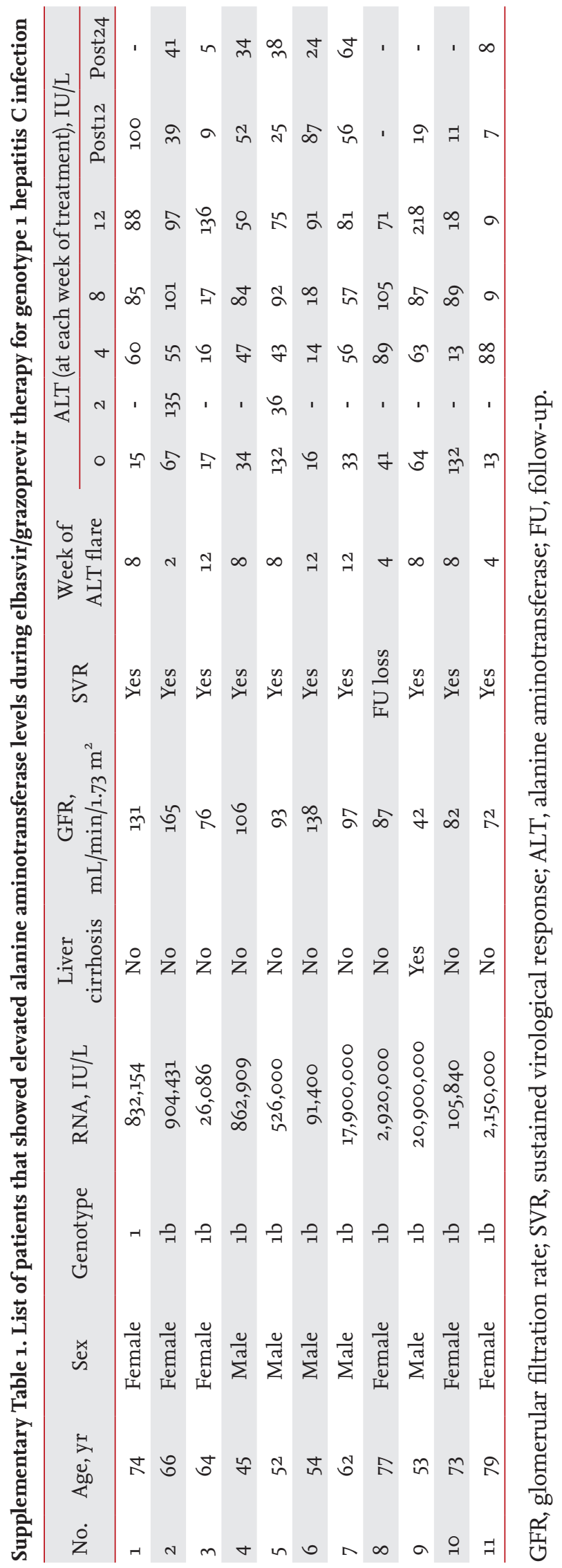

